\title{
Eye-state-find: Sleepy or Yawn Driver Detection using Facial Features Extraction with Classification
}

\author{
Sarath Kumar S, Mani. V
}

\begin{abstract}
Tiredness and weariness of car drivers lessen the drivers' capacities of vehicle control, characteristic reflex, acknowledgment and observation. Such decreased cautiousness dimension of drivers is seen around evening time driving or overdriving, causing mishap and posture extreme danger to humanity and society. In this manner it is particularly important in this ongoing pattern in vehicle industry to consolidate driver help framework that can recognize tiredness and weakness of the drivers. This undertaking presents a nonintrusive model PC vision framework for checking a driver's carefulness progressively. Eye following is one of the key advancements for future driver help frameworks since human eyes contain much data about the driver's condition, for example, look, consideration level, and weakness level. One issue regular to many eye following techniques proposed so far is their affectability to lighting condition change. This will in general fundamentally limit their degree for car applications. Ongoing recognition and following of the eye is a functioning territory of research in PC vision network. Restriction and following of the eye can be valuable in face arrangement. This undertaking portrays ongoing eye identification and following technique that works under factor and sensible lighting conditions. It depends on an equipment framework for the continuous obtaining of a driver's pictures utilizing camera and the product execution for checking eye that can maintain a strategic distance from the mishaps.
\end{abstract}

Index Terms: Electro encephala gram, Image processing, Drowsiness, Vehicle control, Driver detection.

\section{INTRODUCTION}

Recent studies prove that road accidents occur mostly due to driver's ill health and inattention so health care monitoring system for drivers is given more importance in order to reduce increasing accidents. It will be useful if smart health care monitoring system is available at an affordable price. Rest is a noteworthy occasion of our day by day lives. Its quality comprises a basic pointer of individuals' wellbeing conditions, both rationally and physically. Existing sensor-based or vision-based rest checking frameworks either is obstructive to utilize or neglect to give sufficient inclusion. With the quick extension of remote foundations these days, channel information, which is inescapable and straightforward, rises as another option. It is very much perceived that these days the rest related medical issues could

Revised Manuscript Received on 20 September, 2019.

SarathKumar.S, PG Student, Department of Computer Science and Engineering, M.Kumarasamy College of Engineering Karur, Tamilnadu, India, skysarathkumar@gmail.com

Mani.V, Assistant Professor, Department of Computer Science and Engineering, M Kumarasamy College of Engineering Karur, Tamilnadu, India, maniv.cse@mkce.ac.in eat up much general wellbeing asset and force substantial weight on the administration spending plan. Driver safety monitoring is not only important for fleet management, but also for monitoring new drivers and assessing performance of drivers during training sessions. Driver security can be surmised from driver style, which is naturally delegated either average (non-forceful) or forceful. So as to defeat the surprising expense of these business frameworks, we have made a novel application for both deciding a driver's style (non-forceful versus forceful), just as perceiving kinds of driving occasions utilizing just the sensors on a cell phone. As opposed to past research on driving occasion acknowledgment, our framework is actualized totally on a cell phone without utilizing outer equipment for preparing or audit.

\section{RELATED WORK DRIVER MONITORING}

Sleepiness during driving is a major cause for road accidents. Road accidents are undoubtedly becoming a growing concern in many countries because they are rising to become one of the leading causes of death and injuries. Most people thought that drunken driving is the serious cause of accidents and unaware of drowsy driving which is just fatal. It also deteriorates vigilance, concentration and alertness so that the ability to perform different consciousness-based activities (such as driving) is impaired, decreases awareness, reduces judgment and increases the risk of crashing. Road accidents caused due to driver fatigue is more serious and leads to death other than drunken driving and rush driving accidents due to drowsiness is more crucial because the driver is loss the consciousness which leads to serious injuries or death. Not only are the people traveling in vehicles the victims. The pedestrians will also get affected. The cause of a fatal crash where drowsy driving involves is nearly impossible to determine with certainty. However, the investigators tell that there are a number of clues at a crash scene that shows the person fell asleep at the wheel. For example, accidents due to drowsy driving occurs usually in vehicles where the driver is alone and the injuries seems to be serious or fatal, especially during nights the drivers drive under stress on highways and as a result, they mostly lose control over the vehicle and become the victims of accidents. Classification of driver behaviour considered as a complex issue because it is a multi-dimensional problem and is subjected to several peculiarities of driver and traffic state The traffic state is derived by group of variables like road conditions, vehicle kinematic and driver behaviour. 


\section{SLEEPING MONITORING}

PSG, BCG, PPG and ACT are four standard techniques for the conventional resting observing frameworks. PSG is known for its rich and precise physiological parameters like cerebrum action (electroencephalogram, EEG), eye developments (electrooculography, EOG), cardiovascular capacity (electrocardiogram, ECG), and so on. The broadly received PSG is the most solid test and fills in as the brilliant standard in genuine world. In any case, PSG is generally performed in a rest research facility via prepared specialists. Subsequently usually blamed for being tedious and costly. BCG measures the ballistic powers on the heart. It speaks to an indispensable body that is especially helpful for checking the physical status of a person in rest. The typical method to gauge BCG is to append weight sensors to objects supporting the human body, similar to a seat or a sleeping pad. BCG can be performed at home where individuals feel comfortable, and hence is appropriate for long haul assessment. Be that as it may, its transportability remains an issue. PPG and ACT are convenient. PPG utilizes LED sensors to gauge blood vessel oxygen immersion or pulse while ACT utilizes smaller than expected accelerometers to record the action information. They both can be installed into a wrist-watch-like gadget bore. A few such business items are as of now accessible available, for example, MI Band and Fit bit. PSG, BCG, PPG and ACT assess the rest status of an individual from alternate points of view. In any case, there is one regular component they share: depending on intrusive or contact sensors. It raises worries about comfort and convenience. Along these lines, specialists start to investigate the likelihood of dozing screen from a remote place. One conceivable applicant is the vision-based framework. Be that as it may, rest commonly occurs in a low-light condition where infrared or warm cameras, which are expensive, are favoured. Also, the protection issue is another significant concern. To this end, the remote flag that is straightforward to clients rises as another worldview for rest observing in a "clairvoyant" manner.

\section{WIFI-BASEDGESTURE RECOGNITION}

It is outstanding that human nearness meddles the remote flags due to multipath and blurring impacts. Be that as it may, just as of not long ago such meddles have been investigated for movement acknowledgment. Channel reaction information is basic for catching the flag mutilation brought about by human nearness or movements. Past research mostly centres on particular equipment like Radio Frequency (RF) sensors, Doppler radar and Software Defined Radio (SDR) to acquire the fine-grained channel reaction. For example, Kim et al. proposed a pulse recognition framework amid rest utilizing three kinds of Radio Frequency (RF) sensors on wrist, i.e., a solitary resonator, the exhibit resonators, and an infusion bolted PLL resonator sensor. Boboli et al. structured noncontact physiological radar observing framework comprising of two ceaseless wave Doppler radars at $2.45 \mathrm{GHz}$. These frameworks exhibited the possibilities of utilizing remote signs for rest checking. In any case, their practicability and versatility are restricted in genuine applications. Then again, the financially savvy Wi-Fi foundation is generally available these days. The most usually utilized indictor for the channel reaction of Wi-Fi is the Received Signal Strength (RSS), a coarse-grained control include summed over all engendering ways. Sigg et al. were among the first to investigate RSS for perceiving hand movements. Afterward, GU et al. demonstrated that RSS is likewise pertinent for the entire body movements like strolling and running. RSS is convenient, however unfit when managing the multipath impact. Along these lines, Channel State Info (CSI), which describes the remote signs with the recurrence, adequacy (vitality highlight) and stage data, before long comes in as a superior option. Zeng et al. supplanted RSS with CSI in perceiving hand movements and accomplished better execution.

\section{SENSOR BASED IMPLEMENTATION}

There are many medical bio sensors which detects particular values so these sensors are placed on the human body and collect the necessary readings from the human body. Then the information collected is sent to the main controller. This main controller is responsible for transmitting human's health status to the required locations. The wearable sensors used in this model are Heart beat can be sensed by using a high intensity type LED \&LDR. The finger is placed between LED \&LDR. Photodiodes or phototransistors can be used as sensors. The skin maybe illuminated with visible red light using transmitted or reflected light for detection.

\section{LITERATURE REVIEW}

[1]With the improvement of distributed computing, distributed computing based versatile applications, for example, continuous video gushing, constant face acknowledgment have turned out to be mainstream on-going years. Besides, a brought together cloud is difficult to deal with the different administration demands from billions of portable clients. In addition, distributed computing brought together server farms are deficient in adaptability and unfit to help portability for versatile clients. To take care of the issue, CISCOR proposed haze figuring (FC) design even at $10 \%$ CPU use, the devoured power surpasses half of pinnacle control. Therefore, the nature of correspondence association is updated, which further decreases the correspondence delay between versatile clients and the hubs. In this paper, we structure and execute a holder relocation administrator model framework, where application assignments are laid in migratable compartments and situated in hubs. Also, we consider the holder movement in FC as a stochastic improvement issue.

[2]A large number of people are slaughtered each year around the globe in the street mishaps. As indicated by the World Health Organization (WHO) surveys certainty sheet on street traffic wounds, around 1.25 million individuals pass on because of street car accidents every year (3400 passing's for each day). It is the expectation of such advances that nations, for example, Japan and Sweden have openly declared a target of coming to -zero traffic casualty social orders by 2020. Shrewd Transportation Society of America (ITSA) condenses its central goal presentation as "vision zero" which means its goal is to 
diminish the deadly mishaps and deferrals however much as could be expected. V2V correspondence enables vehicles to speak with one another and to share data in regards to their state (e.g., position, speed, increasing speed, and so forth.) or data about the traffic (e.g., condition of traffic lights, mishaps, roads turned parking lots, the line works, and so on.). In any case, V2I correspondence permits the participation between street framework and vehicles. In addition, VANETs are utilized to help security basic applications and non-wellbeing infotainment or stimulation based applications.[3] Motion acknowledgment has as of late been a hotly debated issue in the scholarly community and industry for enormously advancing Human-Computer Interface (HCI). It empowers clients to pass on directions to collaborate with gadgets helpfully just by performing motions, and in this manner is extensively connected in our day by day life. For instance, in a shrewd house, individuals can remotely control family unit types of gear, for example, $\mathrm{TV}$, forced air system, fridge, by doing straightforward signals with no extra controller. Vision-based signal acknowledgment frameworks make utilization of cameras and PC vision procedures to perceive motions. Sensor-based frameworks utilize different sorts of sensors as motions input interface, however expect clients to wear gadgets with them. Basically, a signal acknowledgment process is a mapping procedure from the physical world to the computerized world. Along these lines, extricating computerized highlights of each signal, spoken to by CSI values, is an essential for this mapping procedure. Despite the fact that CSI is relied upon to display diverse changes to signals, it is non-paltry to remove the relating highlights from these loud CSI changes to depict motions in the physical world.[4]The previous couple of years have seen a flood of enthusiasm for pervasive wellbeing observing. Today, we see savvy homes that constantly screen temperature and air quality and utilize this data to enhance the solace of their occupants. As wellbeing observing advances advance further, we imagine that future shrewd homes would screen our condition, as well as screen our imperative signs, such as breathing and pulses. We depict the activity of Vital-Radio and show through a client think about that it can follow clients' breathing and pulses with a middle precision of $99 \%$, notwithstanding when clients are 8 meters from the gadget, or in an alternate room. Moreover, it can screen the essential indications of various individuals at the same time. Further, a client approaching a Vital-Radio empowered stand in a new area, (for example, an air terminal) may get altered help dependent on his feeling of anxiety.[5]The expanding number of auto collisions because of a decreased driver's watchfulness level has turned into a difficult issue for society. Insights demonstrate that somewhere in the range of $10 \%$ and $20 \%$ of all the auto collisions are because of drivers with a reduced cautiousness level. Moreover, mishaps identified with driver hypo-watchfulness are more genuine than different sorts of mishaps, since lethargic drivers frequently don't make equivocal move preceding a crash. Consequently, creating frameworks for checking a driver's dimension of watchfulness and cautioning the driver, when he is sluggish and not giving careful consideration to the street, is basic to forestall mishaps. The strategies for weariness identification basically centred around proportions of the driver's state, driver execution and the blend of the driver's state and execution. The proportions of driver's state included PERCLOS, mouth shape and head position; the proportions of driver execution included path following and following of separation between vehicles. These methodologies are introduced and examined in detail. Some regular driver checking frameworks are additionally presented in this paper.[6]Remote Sensor Network's (WSN) establishes of substantial number of sent sensor hubs and base hubs. Base hubs gather the information from sent sensor hubs. Each conveyed sensor hub is outfitted with a battery gadget and all in all they are sent arbitrarily. Thus the area of each sent hub is obscure. Therefore, calculations are created to understand the working of WSN's without knowing the correct area of transmitting hub. The situation of conveyed sensor hubs can be found utilizing both of Time of Arrival (TOA), Time Difference of Arrival (TDOA) or Received Signal Strength (RSS) calculations. In this paper we attempted to evaluate the inexact separations of sent sensor hubs utilizing mean RSS estimations, from which the situation of conveyed sensor hub can be found. Consequently we presume that, under perfect conditions by utilizing advanced guess calculations, productive limitation can be accomplished through RSSI estimations. Later on we will endeavour to limit the conveyed sensor hubs on two dimensional and three measurement plane utilizing mean RSSI values.[7] Weariness is a steady word related risk for any long separation or expert driver, and can influence one's judgment of his or her appropriateness to keep driving. Productivity and execution can be impeded amid weakness when an individual holds on in proceeding with the present movement as ordinary. Lamont and Dawson revealed that a driver who has stayed without rest for $24 \mathrm{~h}$ has diminished driving aptitudes, and is equivalent to driving with wrongfully high blood liquor convergence of $0.10 \%$. Exhaustion is free of vitality utilization and can't just be estimated by execution weakness. Consequently, the requirement for physiological weakness countermeasures emerges to avert exhaustion related mishaps. Terrific Prix was utilized as driving test system programming. The video show from the product demonstrated different vehicles, the driving condition and the present speed and other street boosts. Following the alarm driving session was the dreary driving session, in which members were required to drive ceaselessly somewhere in the range of 60 and $80 \mathrm{~km} / \mathrm{h}$ for around $1 \mathrm{~h}$. Synchronous physiological estimations were recorded amid the driving sessions. The NeuroScan framework was utilized to record the physiological information.[8]Crucial signs, for example, breathing rate and pulse, demonstrate the condition of an individual's basic body capacities. They are the fundamental parts to evaluate the general physical soundness of an individual and distinguish different infection issues. For instance, F. Adib et al. use Universal Software Radio Peripheral (USRP) and Frequency Modulated Continuous Wave (FMCW) radar to screen an individual's breathing rate by distinguishing the chest vacillations brought about by relaxing. Doppler radar and ultra-band radar are used to find an individual's breathing separately. These frameworks include specific gadgets with high multifaceted nature, which keep them from huge scale 
and long haul arrangement. Their methodology requires extra remote system framework (i.e., devoted sensor hubs), and the coarse-grained channel data can't catch the crucial indications of pulse. Another new heading is utilizing wearable sensors, (for example, Fitbit and Jawbone ) to follow individuals' wellness whenever. Yet, they just have the capacity of performing coarse-grained rest observing without catching the breathing rate, which is basic to many rest issue finding including rest apnea.[9]With the quick advancement of universal detecting innovations and quick development of the world maturing populace, astute conditions that can screen and respond to older folks' every day exercises have gotten a great deal of consideration. While early work concentrated basically on condition checking and day by day movement acknowledgment, the previous couple of years have seen a flood of enthusiasm for wellbeing danger observing to guarantee opportune mediation and security for older folks, for example, fall recognition and fundamental sign checking. So as to limit the inconvenience brought by the intrusive breath estimation strategies, a few endeavours have been made utilizing wearable and weight sensors for long haul breath checking. While these two sorts of contact-based detecting strategies are progressively passable for older folks, the weight sensor based frameworks won't have the capacity to gauge breath rate when the subject leaves the bed, while wearable breath estimation gadgets have the issues of acknowledgment or convenience for a significant number of seniors. Driven by the above inquiries, in this paper, we present a novel WiFi Fresnel demonstrate and build up the related hypothesis to support the hypothetical establishment for WiFi radio engendering in indoor condition.[10]The expanding number of auto collisions because of a reduced driver's carefulness level has turned into a difficult issue for society. Measurements demonstrate that $20 \%$ of all the car crashes are because of drivers with a lessened cautiousness level. Besides, mishaps identified with driver hypo-carefulness are more genuine than different sorts of mishaps, since lethargic drivers frequently don't make right move preceding an impact. Consequently, creating frameworks for checking driver's dimension of watchfulness and cautioning the driver, when he is tired and not giving careful consideration to the street, is basic to anticipate mishaps. Driver's condition of carefulness can be portrayed by driver execution with an emphasis on the vehicle conduct or dependent on driver's physiological/physical state. Programmed acknowledgment (or investigation) of outward appearance comprises of three dimensions of assignments: confront recognition, outward appearance data extraction, and articulation grouping. In these assignments, the data extraction is the primary issue for the element based outward appearance acknowledgment from a picture arrangement. It includes location, distinguishing proof and following facial component focuses under various enlightenments, confront introductions and outward appearances.

\section{MACHINE LEARNING AND ALGORITHMS}

\section{A. Convolutional Neural Network}

Convolutional Neural Network (CNN) in Deep learning strategy is best technique which is multilayer neural system with convolutional channel to change the info picture by preparing to order under persuaded classes to some degree.
There are exemplary and current building structures are available in $\mathrm{CNN}$ for picture investigation [11]. In $\mathrm{CNN}$, there are 3 layers where convolutional layer, max pooling layer, fully connected layer. Because of having three layer the image can be extracted with high resolution than the other networks where each Layer acknowledges an info 3D volume and changes it to a yield $3 \mathrm{D}$ volume through a differentiable capacity.

\section{B.HAAR-LIKE Features}

Haar-like highlights are computerized picture highlights utilized in item acknowledgment. They owe their name to their natural comparability with Haar wavelets and were utilized in the primary continuous face identifier. Generally, working with just picture forces (i.e., the RGB pixel esteems at every single pixel of picture) made the undertaking of highlight estimation computationally costly. [12]A Haar-like component thinks about neighboring rectangular locales at a particular area in an identification window, totals up the pixel forces in every district and ascertains the contrast between these aggregates. This distinction is then used to sort subsections of a picture. For instance, let us state we have a picture database with human appearances. It is a typical perception that among all faces the locale of the eyes is darker than the district of the cheeks. The situation of these square shapes is characterized in respect to a recognition window that demonstrations like a jumping box to the objective article (the face for this situation). The key preferred standpoint of a Haar-like element over most different highlights is its figuring speed. Because of the utilization of necessary pictures, a Haar-like element of any size can be determined in consistent time (roughly 60 microchip guidelines for a 2-square shape highlight).

\section{C.K- Nearest Neighbour Classification}

In eye state acknowledgment, the KNN calculation is a strategy for ordering objects dependent on nearest preparing models in the element space. KNN is a kind of case based learning, or lethargic realizing where the capacity is just approximated locally and all calculation is conceded until order. [13] The $\mathrm{KNN}$ is the principal and most straightforward arrangement strategy when there is practically zero earlier information about the conveyance of the information. This standard essentially holds the whole preparing set amid learning and appoints to each inquiry a class spoken to by the greater part mark of its k-closest neighbours in the preparation set. The Nearest Neighbour rule $(\mathrm{NN})$ is the easiest type of $\mathrm{KNN}$ when $\mathrm{K}=1$. In this technique each example ought to be ordered correspondingly to its encompassing examples. Along these lines, on the off chance that the order of an example is obscure, it could be anticipated by considering the characterization of its closest neighbour tests. [14]Given an obscure example and a preparation set, every one of the separations between the obscure example and every one of the examples in the preparation set can be figured. The separation with the littlest esteem relates to the example in the preparation set nearest to the obscure example. Consequently, the obscure example might be characterized dependent on the order of this closest neighbour.

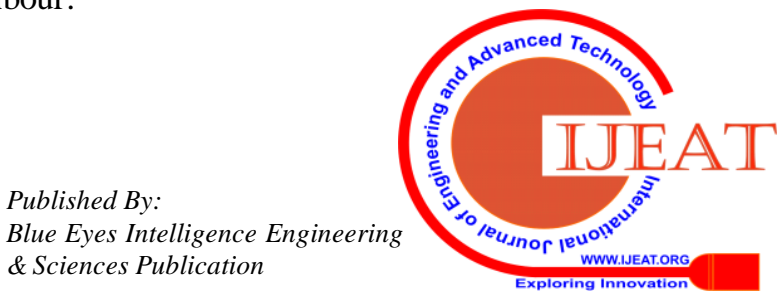




\section{Rectangular HAAR like features}

A basic rectangular Haar-like element can be characterized as the distinction of the entirety of pixels of zones inside the square shape, which can be at any position and scale inside the first picture. This altered list of capabilities is called 2-square shape include. Viola and Jones additionally characterized 3-square shape highlights and 4-square shape highlights. The qualities demonstrate certain attributes of a specific territory of the picture. Each component type can show the presence (or nonappearance) of specific qualities in the picture, for example, edges or changes in surface.

\section{E. Course Classifier}

The course classifier comprises of a rundown of stages, where each stage comprises of a rundown of powerless students. The framework identifies protests being referred to by moving a window over the picture. Each phase of the classifier names the particular locale characterized by the present area of the window as either positive or negative positive implying that an item was found or negative implies that the predetermined article was not found in the picture. In the event that the naming yields a negative outcome, the order of this particular area is therefore finished and the area of the window is moved to the following area. On the off chance that the naming gives a positive outcome, the area moves of to the following phase of arrangement. The classifier yields a last decision of positive, when every one of the stages, including the last one, yield an outcome, saying that the item is found in the picture. A genuine positive implies that the item being referred to is for sure in the picture and the classifier marks it thusly - a positive outcome. A false positive implies that the marking procedure erroneously establishes that the item is situated in the picture, despite the fact that it isn't. A false negative happens when the classifier can't distinguish the real article from the picture and a genuine negative implies that a non-object was accurately classifier as not being the item being referred to. So as to function admirably, each phase of the course should have a low false negative rate, supposing that the genuine item is named a non-object, at that point the arrangement of that branch stops, with no real way to address the error made. Be that as it may, each stage can have a generally high false positive rate, on the grounds that regardless of whether the n-th arrange orders the non-object as really being the article, at that point this mix-up can be settled in $n+1-$ th and consequent phases of the classifier.

\section{CONCLUSION}

Drowsiness and fatigue of automobile drivers reduce the drivers' abilities of vehicle control, natural reflex, recognition and perception. Such diminished vigilance level of drivers is observed at night driving or overdriving, causing accident and pose severe threat to mankind and society. The proposed system can be used for driver's safety and its consequences. The system detects drowsiness of driver through eye conditions. It based on face detection using well known Viola Jones algorithm, eyes are detected through proposed crop Eye algorithm which segments the face in different segments in order to get left and right eye. The proposed method was tested in video sequence recorded in vehicle as well as in lab environment. The proposed system works in real time with minimal computational complexity. Therefore it is also suitable for implementing in surveillance environment. The system produces $90 \%$ accurate results for different faces. However, its limitation is detecting the eyes of person wearing glasses. Also it does not produce accurate results if any reflective object is found behind the driver. In future, we can consider the limitations and implemented with embedded system.

\section{REFERENCES}

1. B. T. Jap, S. Lal, P. Fischer, and E. Bekiaris, "Using eeg spectral components to assess algorithms for detecting fatigue," Expert Systems with Applications, vol. 36, no. 2, pp. 2352-2359, 2009.

2. P. Kumar, L. Reddy, and S. Varma, "Distance measurement and error estimation scheme for rssi based localization in wireless sensor networks," in Wireless Communication and Sensor Networks (WCSN), 2009 Fifth IEEE Conference on. IEEE, 2009, pp. 1-4

3. [3] W. He, K. Wu, Y. Zou, and Z. Ming, "Wig: Wifi-based gesture recognition system," in Computer Communication and Networks (ICCCN), 2015 24th International Conference on. IEEE, 2015, pp. 1-7.

4. V.Mani, K.Nivetha, M.Satheeswaran, K.M.Sendurrajan, "Privacy Location Sharing Services For Social Networks", 2016 IJPAM.

5. Q. Wang, J. Yang, M. Ren, and Y. Zheng, "Driver fatigue detection: a survey," in Intelligent Control and Automation, 2006. WCICA 2006. The Sixth World Congress on, vol. 2. IEEE, 2006, pp. 8587-8591.

6. Dr.S.Thilagamani, Dr.P.Santhi, "A Survey on Audit Free Cloud Storage Via Deniable Attribute Based Encryption”, vol.05, No.1, 2016.

7. J. Liu, Y. Wang, Y. Chen, J. Yang, X. Chen, and J. Cheng, "Tracking vital signs during sleep leveraging off-the- shelf wifi," in Proceedings of the 16th ACM International Symposium on Mobile Ad Hoc Networking and Computing. ACM, 2015, pp. 267-276.

8. H. Wang, D. Zhang, J. Ma, Y. Wang, Y. Wang, D. Wu, T. Gu, and B. $\mathrm{Xie}$, "Human respiration detection with commodity wifi devices: do user location and body orientation matter?" in Proceedings of the 2016 ACM International Joint Conference on Pervasive and Ubiquitous Computing. ACM, 2016, pp. 25-36.

9. M. Saradadevi and P. Bajaj, "Driver fatigue detection using mouth and yawning analysis," International journal of Computer science and network security, vol. 8, no. 6, pp. 183-188, 2008.

10. S.Saravanan,Arivarasan(2014)."Anefficient ranked Keyword search for effective utilization of outsourcedclouddata" Journal of Global Research in Computer Science, Vol4(4), pp:8-12

11. S Saravanan, V Venkatachalam(2016) “' Improving map reduce task scheduling and micro-partitioning mechanism formobile cloud multimedia services" International Journal of Advanced Intelligence Paradigms, Vol 8(2),pp157- 167.

12. S Saravanan, V Venkatachalam(2014) ,"Advance Map Reduce Task Scheduling algorithm using mobile cloud multimedia services architecture" IEEE Digital Explore,pp21-25.

13. S.Swathi(2015) "Preemptive Virtual Machine Scheduling Using CLOUDSIM Tool”, International Journal of Advances inEngineering, 1(3), 323 -327 ISSN: 2394-9260, pp:323-327.

14. S Saravanan, V Venkatachalam, S Then Malligai(2015) "Optimization of SLA violation in cloud computing using artificial bee colony", 1(3), 323 -327 ISSN: 2394-9260, pp:410-414. 